\title{
The Trend of Dental Check-Up And Incidence of Dental Complications Following The Use of Bone Modifying Agents In Patients With Metastatic Breast And Prostate Cancer: Analysis of Data From The Korean National Health Insurance Service
}

Ah Reum Lim

Korea University Anam Hospital

Wonse Park

Yonsei University

Seok Joo Moon

Korea University College of Medicine

Min Sun Kim

Korea University College of Medicine

Soohyeon Lee ( $\sim$ soohyeon_lee@korea.ac.kr)

Korea University Anam Hospital https://orcid.org/0000-0001-9665-062X

\section{Research Article}

Keywords: Metastatic breast cancer, Metastatic prostate cancer, Bone metastasis, Dental check-up, Medication-related osteonecrosis of the jaw

Posted Date: January 14th, 2022

DOI: https://doi.org/10.21203/rs.3.rs-1174780/v1

License: @ (i) This work is licensed under a Creative Commons Attribution 4.0 International License.

Read Full License 


\section{Abstract}

Purpose: Bone-modifying agents (BMAs) are key components in the management of cancer patients with bone metastasis. Despite their clinical benefits, the use of BMAs is associated with adverse dental events including medication-related osteonecrosis of the jaw (MRONJ). This study investigated the rate of preoperative dental surveillance to reduce the incidence of adverse dental events including MRONJ after BMA treatment in patients with bone metastasis from breast and prostate cancer.

Methods: Data, including age, cancer diagnosis, administered BMAs, and adverse dental events during cancer treatment, of patients with bone metastasis from breast and prostate cancer who received at least one infusion of BMA between 2007 and 2019 were extracted from the Korean National Health Insurance Service (KNHIS) dataset.

Results: Of the 15357 patients who received BMAs, 1706 patients (11.1\%) underwent dental check-ups before BMA treatment. The proportion of patients receiving dental check-ups increased from $4.4 \%$ in 2007 to $16.7 \%$ in 2019. Referral for dental check-up was more frequent in clinics and primary hospitals than in tertiary hospitals, and from the departments of internal medicine and urology than from the department of general surgery, regardless of the patient's health insurance status. After BMA treatment, 3328 patients (21.6\%) developed adverse dental events, including tooth extraction (73.0\%), abscess (16.9\%), acute periodontitis (5.3\%), acute pericoronitis (2.6\%), and MRONJ (2.2\% of $3328,0.5 \%$ of 15357$)$.

Conclusions: Considering the long treatment period in patients with metastatic cancer, coordination between dentists and oncologists is necessary to ensure appropriate dental treatment before the initiation of BMAs.

\section{Introduction}

The use of bone-modifying agents (BMAs), such as bisphosphonates and receptor activation of nuclear factor kappa-B (RANK) ligand inhibitors, in patients with bone metastases from breast and prostate cancer is associated with improvements in morbidity, pain, quality of life, and skeletal-related events (SREs). BMAs are also recommended in patients with advanced lung cancer, renal cancer, and other solid tumors with bone metastases, particularly those at high risk of SREs and a life expectancy $>3$ months [1].

Despite several clinical benefits, the use of BMAs is associated with medication-related osteonecrosis of the jaw (MRONJ), which occurs in approximately $1-9 \%$ of patients with advanced cancer [2-5]. MRONJ can occur spontaneously, or following invasive dental procedures such as tooth extraction, and its signs and symptoms include exposure of the maxilla, pain due to inflammation of the exposed area, bleeding, osteonecrosis of the inferior alveolar canal and maxillary sinuses, and orodermal fistula. MRONJ can cause serious functional and masticatory disorders with a significant influence on patient quality of life, delay in scheduled cancer treatment, and death [6]. 
With increase in the overall survival and treatment period of cancer patients with bone metastasis, the incidence of adverse dental events, including MRONJ, has increased. During the long period of treatment for metastatic cancer, chronic dental infections such as periodontitis, periapical lesions, or pericoronitis caused by wisdom teeth can be converted into acute dental infections due to decreased immune function. Acute dental infection can cause pain, swelling, bleeding, and sometimes require invasive treatment, such as tooth extraction, during chemotherapy. This often leads to the discontinuation or delay of chemotherapy during dental care [7].

Therefore, the European Society for Medical Oncology (ESMO), the American Society for Clinical Oncology (ASCO) and Cancer Care Ontario (CCO) published guidelines on the use of BMAs in metastatic bone disease and recommended that oral care assessment, including a comprehensive dental, periodontal, and oral radiographic examination should be undertaken before initiating therapy $[1,8]$. Many studies have established that preventive oral care methods combined with effective oral health practices are associated with a lower incidence of MRONJ [9-11].

Although it is necessary to establish a dental care plan and to ensure that medically essential dental procedures are performed through coordination between dentists and oncologists prior to initiating BMA treatment, dental referral and surveillance are not actively practiced. This may be related to the perception that anti-cancer therapy is more critical than dental evaluation and a fear of the excessive cost of dental treatment or dental procedure-induced pain [12]. According to a systematic review by Hong et al. [6, 7], the incidence of dental infection during chemotherapy is approximately $5.4-5.8 \%$. However, most studies have been reported from one or several institutions. Therefore, there are very few studies on the incidence of dental adverse events including MRONJ during chemotherapy on a national level.

We aimed to investigate the rate of dental check-ups before BMA treatment and occurrence of adverse dental events including MRONJ after BMA treatment in patients with bone metastasis from breast and prostate cancer using claims data from the Korean National Health Insurance Service (KNHIS) database.

\section{Material And Methods}

\section{Data source}

The KNHIS enrolls more than $97 \%$ of the Korean population. We used KNHIS claims data from January 2007 to December 2019. These data provide detailed information on demographics and healthcare utilization, including diagnostic codes (International Classification of Disease 10th revision, ICD-10), procedure codes, and prescriptions.

\section{Study population}

We identified all newly diagnosed patients with breast and prostate cancer between January 2007 and December 2019 (ICD-10 codes: C50 and C61). We used a 6-month washout period to exclude cancer patients who had been diagnosed with bone metastasis in the past. Patients were followed up for at least 
2 years and assessed for the development of bone metastases and adverse dental events. Patients diagnosed with bone metastases were defined as those with at least three claims per year for the prescription of BMAs, such as denosumab, zoledronic acid, and pamidronate, using drug prescription codes (ATC codes M05BX04, M05BA08, and M05BA03). Adverse dental events were identified using the procedure codes for irrigation and drainage (U4454-U4457, U4464, U4467) and tooth extraction (U4411U4417, U4420, UD620), and diagnostic codes for dental complications such as inflammatory conditions of the jaw (MRONJ), acute periodontitis, periodontal abscess of gingival origin, and acute pericoronitis using a (ICD-10 codes (K052, K0520-K0522, K0528, K0529, K102, and M871). Dental check-ups were identified using the EDI codes for clinical oral evaluations (AA100, AA106, AA107, AA109 AA200, AA206, AA207, AA209), panoramic radiographic images (G9701), and procedure codes for scaling (U2233).

\section{Statistical analysis}

Data on the incidence of dental check-ups and adverse dental events in each type of cancer and the basic demographic characteristics of the patients were obtained. Demographic characteristics were summarized as mean (standard deviation) and range (minimum, maximum) for continuous variables and frequency (percentage) for categorical variables. The incidence of adverse dental events was analyzed, and the annual incidence was also presented. The annual incidence of MRONJ (ICD-10 code K102) was investigated using the prescription codes for pamidronate and zoledronic acid. Proportions of dental care were summarized according to patients' residential area, hospital type, and types of medical benefits. Chi-squared tests were performed to compare the proportions of complications and medical departments between the group that received dental care and the group that did not. All statistical analyses were performed using SAS software (version 9.4; SAS Institute Inc., Cary, NC, USA), and statistical significance was set at two-tailed $p<0.05$.

\section{Results}

\section{The trend of dental check-up before BMA administration}

A total of 15357 patients with breast or prostate cancer who developed bone metastasis were treated with BMA between 2007 and 2019. Of 15357 patients, the number of male and female patients was 3643 (23.7\%) and 11714 (76.2\%), respectively. Among the male patients, $3584(98.4 \%)$ had prostate cancer and $59(1.6 \%)$ had breast cancer. The median ages of men and women were 72 years and 53 years, respectively (Supplementary Table 1). According to the KNHIS database, the proportion of patients who received dental check-ups among those treated with BMAs for breast or prostate cancer with bone metastases increased from 3.1\% in 2007 to $8.3 \%$ in 2015, decreased to $3.8 \%$ in 2016, and then increased again to $11.4 \%$ in 2018 (Supplementary Table 2).

Overall, of the 15357 patients, 1706 (11.1\%) underwent dental check-ups prior to BMA treatment. The proportion of patients undergoing dental check-ups steadily increased from $4.38 \%$ in 2007 to $16.72 \%$ in 2019 (Fig. 1). 


\section{Dental check-up rate according to hospital type and department}

We analyzed dental check-up status prior to BMA treatment based on the type of hospital, department, and health insurance (Table 1). The proportion of dental surveillance before BMA treatment showed a difference according to the size of the hospital; it was the highest at clinics/ primary hospitals (17.3\%), followed by general hospitals (12.2\%), and tertiary general hospitals (9.9\%).

Further, there was a difference in the dental referral rate according to the department-11.1\% from internal medicine, $13.3 \%$ from urology, and $9.3 \%$ from general surgery. In terms of health insurance status, there was no significant difference in the proportion of patients who underwent dental check-up between those covered by health insurance $(11.1 \%)$ and others (medical care, homeless people, foreign workers $[11.5 \%])$.

The comparison of dental check-ups status by region showed that mid-sized cities, such as Gwangju (15.3\%), Daejeon (14.1\%), and Ulsan (13.1\%) had a higher proportion of patients with dental check-ups before BMA treatment than Seoul (11.0\%) and Inchon (8.1\%). Jeollabuk-do (8.8\%) and Gyeongsangbukdo $(5.9 \%)$ reported dental check-up rates far below the average; thus, it is necessary to improve the distribution of medical resources and related services (Fig. 2).

\section{Adverse dental events after BMA treatment}

Adverse dental events occurred in 3328 (21.7\%) of 15357 patients receiving BMA treatment. MRONJ was reported in 74 cases, accounting for $2.2 \%$ of all adverse dental events and $0.5 \%$ of all patients receiving BMAs, which was lower than that reported in pivotal clinical studies. Of 3328 patients with adverse dental events, 2429 (73.0\%) underwent tooth extraction and 309 (9.3\%) underwent incision and drainage treatment. Periapical abscesses of gingival origin without sinus and acute periodontitis occurred in 223 and 175 patients, respectively. Periapical abscesses of gingival origin with sinus occurred in 30 cases, and acute pericoronitis occurred in 88 cases (Table 2 ).

Figure 3 shows the incidence of adverse dental events after BMA treatment. The number of cases was very small in the early phase of the study period, and adverse dental events might not have been completely captured in 2007, but increased every year (Fig. 3). Among 1706 patients who underwent dental check-ups before BMA treatment, 509 (29.8\%) required dental intervention, whereas only 2919 $(20.7 \%)$ of 13651 patients who did not undergo dental surveillance required dental intervention (Table 3 ).

\section{Discussion}

BMAs, such as bisphosphonate and denosumab, are key components in the management of patients with cancer with bone metastases. Bone metastasis may increase morbidity and mortality in patients with a poor prognosis. Although BMAs provide many clinical benefits to prevent SREs, their use is associated with MRONJ, which occurs in approximately $1-9 \%$ of patients with advanced cancer [2-4, 13]. MRONJ can be challenging to treat and can cause significant pain and reduced quality of life. 
Therefore, patients should receive education regarding dental hygiene and MRONJ and undergo a comprehensive dental examination before starting BMA treatment [14]. During the period of BMA use, regular follow-up with a dental professional should be encouraged, but invasive dental procedures should be avoided [15].

To evaluate the adherence to dental guidelines and their practical impact in Korea, we investigated the rate of dental check-up before BMA treatment and adverse dental events after BMA treatment in patients with breast and prostate cancer with bone metastasis using a nationwide database system. Between 2007 and 2019, the overall rate of dental check-ups before BMA treatment was $11.1 \%$, and the proportion increased steadily. Dental surveillance was more actively recommended by the departments of internal medicine and urology than by the department of general surgery and in mid-sized cities than in cosmopolitan areas, including Seoul and Inchon. The frequency of dental check-ups was lower in tertiary general hospitals than that in small hospitals and clinics. Adverse dental events occurred in $3328(21.7 \%)$ of 15357 patients receiving BMA treatment. Of the 3328 patients with adverse dental events, 2429 (73.0\%) underwent tooth extraction and 309 (9.3\%) underwent incision and drainage treatment. MRONJ was reported in 74 cases, accounting for $2.2 \%$ of the 3328 adverse dental events and $0.5 \%$ of all patients receiving BMAs, which appeared to be lower than that reported in pivotal clinical studies. However, the incidence of adverse dental events after denosumab administration was $0.5-2.1 \%, 1.1-3.0 \%$, and $1.3-$ $3.2 \%$ after 1,2 , and 3 years of exposure, respectively. Similarly, a systemic review revealed that the incidence of adverse dental events after zoledronic acid administration was $0.4-1.6 \%, 0.8-2.1 \%$, and $1.0-2.3 \%$ after 1,2 , and 3 years of exposure, respectively [16]. The incidence of adverse dental events after BMA treatment was reported to be $0-0.019 \%$ in clinical trials, which is similar to our findings $[3,17$, 18].

There were some limitations to our study. First, MRONJ was mainly classified using the ICD code K10.2, without considering the clinicopathological aspects. A code for more specific pathological features for MRONJ (M87.1), was introduced in 2010, but errors may have occurred because of the low frequency of its use. Bergdahl et al. reported that osteonecrosis due to drugs (M87.1) had the highest positive predictive value $(83 \%$; $95 \%$ confidence interval, $36-100 \%)$ in a study using data from the Swedish National Patient Registry. Inflammatory conditions of the jaw (K10.2) had a low positive predictive value (16\%) [19]. Therefore, we also analyzed according to codes M87.1 and M87.18, but there was no significant difference between M87.1 and M87.18 in our database. Second, MRONJ may be difficult to diagnose in a dental clinic owing to the nature of dental diseases and lack of experience with MRONJ. Not all patients with dental problems visit dentists. Many patients with metastatic cancer may be reluctant to visit a dentist for economic reasons, owing to cancer treatment schedules, and owing to fear of dental treatment. Medical doctors prescribe pain killers or antibiotics to relieve dental symptoms. These factors may contribute to the low reported rates of dental diagnoses and dental care during BMA treatment in the national claims database system.

Despite these limitations, this is the first study to investigate the incidence of dental check-up before BMA treatment and MRONJ after BMA treatment in a real-world setting. This study was performed using data 
from the nationwide KNHIS database. Therefore, our sample may be considered a representative sample regarding the occurrence of adverse dental events during BMA treatment in patients with bone metastasis from breast and prostate cancer.

We would like to emphasize the importance of collaboration among cancer care teams, oncologists, and dental specialists to prevent adverse dental events to improve the quality of life of patients. For patients with cancer who are scheduled to receive a BMA in a nonurgent setting, oral care assessment, including a comprehensive dental, periodontal, and oral radiographic examination when feasible, should be undertaken before initiating therapy. Based on the assessment, a dental care plan should be developed and implemented with coordination between the dentist and oncologist [15].

\section{Declarations}

Author contribution: Conceptualization: A.R.L., W.P. and S.L.; data acquisition and interpretation: A.R.L., S.J.M. and M.S.K.; formal analysis: A.R.L., S.J.M. and M.S.K.; data curation: A.R.L., S.J.M. and M.S.K.; writing-original draft preparation: A.R.L., W.P. and S.L.; writing-review and editing: A.R.L., W.P. and S.L.; supervision: S.L.. All authors have read and agreed to the published version of the manuscript.

Funding: The authors declare that no funds, grants, or other support were received during the preparation of this manuscript.

Ethics approval: The study was approved by the Institutional Review Board of Korea University Anam Hospital (2020AN0012), and the requirement for written informed consent was waived.

Consent to participate and consent to publish: the Institutional Review Board of Korea University Anam Hospital waived the requirement for written informed consent.

Conflict of interest: The authors have no relevant financial or non-financial interests to disclose.

Data availability: The data presented in this study are available on request from the author.

\section{References}

1. Coleman R, Hadji P, Body JJ et al (2020) Bone health in cancer: ESMO Clinical Practice Guidelines. Ann Oncol 31:1650-1663. https://doi.org/10.1016/j.annonc.2020.07.019

2. Himelstein AL, Foster JC, Khatcheressian JL et al (2017) Effect of Longer-Interval vs Standard Dosing of Zoledronic Acid on Skeletal Events in Patients With Bone Metastases: A Randomized Clinical Trial. JAMA 317:48-58. https://doi.org/10.1001/jama.2016.19425

3. Vahtsevanos K, Kyrgidis A, Verrou E et al (2009) Longitudinal cohort study of risk factors in cancer patients of bisphosphonate-related osteonecrosis of the jaw. J Clin Oncol 27:5356-5362. https://doi.org/10.1200/JCO.2009.21.9584 
4. Stopeck AT, Fizazi K, Body JJ et al (2016) Safety of long-term denosumab therapy: results from the open label extension phase of two phase 3 studies in patients with metastatic breast and prostate cancer. Support Care Cancer 24:447-455. https://doi.org/10.1007/s00520-015-2904-5

5. Qi WX, Tang LN, He AN, Yao Y, Shen Z (2014) Risk of osteonecrosis of the jaw in cancer patients receiving denosumab: a meta-analysis of seven randomized controlled trials. Int J Clin Oncol 19:403-410. https://doi.org/10.1007/s10147-013-0561-6

6. Hong CHL, Gueiros LA, Fulton JS et al (2019) Systematic review of basic oral care for the management of oral mucositis in cancer patients and clinical practice guidelines. Support Care Cancer 27:3949-3967. https://doi.org/10.1007/s00520-019-04848-4

7. Hong CHL, Hu S, Haverman T et al (2018) A systematic review of dental disease management in cancer patients. Support Care Cancer 26:155-174. https://doi.org/10.1007/s00520-017-3829-y

8. Saylor PJ, Rumble RB, Tagawa S, Eastham JA, Finelli A, Reddy PS, Kungel TM, Nissenberg MG, Michalski JM (2020) Bone Health and Bone-Targeted Therapies for Prostate Cancer: ASCO Endorsement of a Cancer Care Ontario Guideline. J Clin Oncol 38:1736-1743. https://doi.org/10.1200/JC0.19.03148

9. Sim le W, Sanders KM, Borromeo GL, Seymour JF, Ebeling PR (2015) Declining Incidence of Medication-Related Osteonecrosis of the Jaw in Patients With Cancer. J Clin Endocrinol Metab 100:3887-3893. https://doi.org/10.1210/jc.2015-1794

10. Walter C, Grotz KA, Kunkel M, Al-Nawas B (2007) Prevalence of bisphosphonate associated osteonecrosis of the jaw within the field of osteonecrosis. Support Care Cancer 15:197-202. https://doi.org/10.1007/s00520-006-0120-z

11. Palaska PK, Cartsos V, Zavras Al (2009) Bisphosphonates and time to osteonecrosis development. Oncologist 14:1154-1166. https://doi.org/10.1634/theoncologist.2009-0115

12. Smith DK, Castellanos EH, Murphy BA (2018) Financial and socio-economic factors influencing preand post-cancer therapy oral care. Support Care Cancer 26:2143-2148. https://doi.org/10.1007/s00520-017-4033-9

13. Coleman RE, Collinson M, Gregory W et al (2018) Benefits and risks of adjuvant treatment with zoledronic acid in stage II/III breast cancer. 10 years follow-up of the AZURE randomized clinical trial (BIG 01/04). J Bone Oncol 13:123-135. https://doi.org/10.1016/j.jbo.2018.09.008

14. Leng S, Lentzsch S (2018) Bone-Modifying Agents: Complicated to Use. J Oncol Pract 14:469-470. https://doi.org/10.1200/JOP.18.00444

15. Yarom N, Shapiro CL, Peterson DE et al (2019) Medication-Related Osteonecrosis of the Jaw: MASCC/ISOO/ASCO Clinical Practice Guideline. J Clin Oncol 37:2270-2290. https://doi.org/10.1200/JC0.19.01186

16. Limones A, Saez-Alcaide LM, Diaz-Parreno SA, Helm A, Bornstein MM, Molinero-Mourelle P (2020) Medication-related osteonecrosis of the jaws (MRONJ) in cancer patients treated with denosumab VS. zoledronic acid: A systematic review and meta-analysis. Med Oral Patol Oral Cir Bucal 25:e326e336. https://doi.org/10.4317/medoral.23324 
17. Coleman R, Woodward E, Brown J et al (2011) Safety of zoledronic acid and incidence of osteonecrosis of the jaw (ONJ) during adjuvant therapy in a randomised phase III trial (AZURE: BIG 01-04) for women with stage II/III breast cancer. Breast Cancer Res Treat 127:429-438. https://doi.org/10.1007/s10549-011-1429-y

18. Ruggiero SL, Dodson TB, Fantasia J, Goodday R, Aghaloo T, Mehrotra B, O'Ryan F, American Association of O, Maxillofacial S (2014) American Association of Oral and Maxillofacial Surgeons position paper on medication-related osteonecrosis of the jaw-2014 update. J Oral Maxillofac Surg 72:1938-1956. https://doi.org/10.1016/j.joms.2014.04.031

19. Bergdahl J, Jarnbring F, Ehrenstein V, Gammelager H, Granath F, Kieler H, Svensson M, Tell GS, Lagerros YT (2013) Evaluation of an algorithm ascertaining cases of osteonecrosis of the jaw in the Swedish National Patient Register. Clin Epidemiol 5:1-7. https://doi.org/10.2147/CLEP.S37664

\section{Tables}

Table 1. Dental check-up by hospital type, department and health insurance

\begin{tabular}{|llll|}
\hline & $\begin{array}{l}\text { Patient who receiving } \\
\text { dental care, } N\end{array}$ & $\begin{array}{l}\text { All } \\
\text { patients, } \\
N\end{array}$ & $\begin{array}{l}\text { Proportion of dental check-up among } \\
\text { all patients (\%) }\end{array}$ \\
Hospital type & & 9858 & 9.9 \\
$\begin{array}{l}\text { Tertiary general } \\
\text { hospital }\end{array}$ & 976 & 4355 & 12.2 \\
General hospital & 532 & 1144 & 17.3 \\
Hospital/clinic & 198 & & \\
Department & 943 & 8507 & 11.1 \\
Internal medicine & 405 & 4379 & 9.25 \\
General surgery & 170 & 1277 & 13.3 \\
Urology & 188 & 1194 & 15.8 \\
Others & 1573 & 1158 & 11.5 \\
$\begin{array}{l}\text { Health insurance } \\
\text { type }\end{array}$ & 133 & 1199 & 1.1 \\
Health insurance & & & \\
Others* & & 1198 & \\
\hline
\end{tabular}

*Person for medical care, homeless people, foreign workers

Table 2. Incidence of adverse dental events during BMA treatment 


\begin{tabular}{|ll|}
\hline Adverse dental events & $N(\%)$ \\
\hline Periapical abscess from gingival origin & \\
\hline Without sinus & $223(6.7)$ \\
\hline With sinus & $30(0.9)$ \\
\hline Acute periodontitis & $175(5.3)$ \\
\hline Acute pericoronitis & $88(2.6)$ \\
\hline Medication-related osteonecrosis of jaw & $74(2.2)$ \\
\hline Treatment & \\
\hline Tooth extraction & $2429(73.0)$ \\
\hline Incision and drainage & $309(9.3)$ \\
\hline Total events & $3328(100.0)$ \\
\hline
\end{tabular}

$B M A$, Bone-modifying agent

Table 3. Dental intervention after the initiation of BMAs

\begin{tabular}{|c|c|c|c|c|}
\hline & $\begin{array}{l}\text { Dental check-up } \\
(N=1706)\end{array}$ & $\begin{array}{l}\text { Without dental check-up } \\
(N=13651)\end{array}$ & p-value* & $\begin{array}{l}\text { * } p \text {-value by chi- } \\
\text { square test }\end{array}$ \\
\hline Dental intervention, $N(\%)$ & & & $<0.0001$ & $M A$, Bone- \\
\hline Yes & $509(29.8)$ & $2819(20.7)$ & & \\
\hline No & $1197(70.2)$ & $10832(79.3)$ & & Fiqures \\
\hline
\end{tabular}




\section{8}

16.7

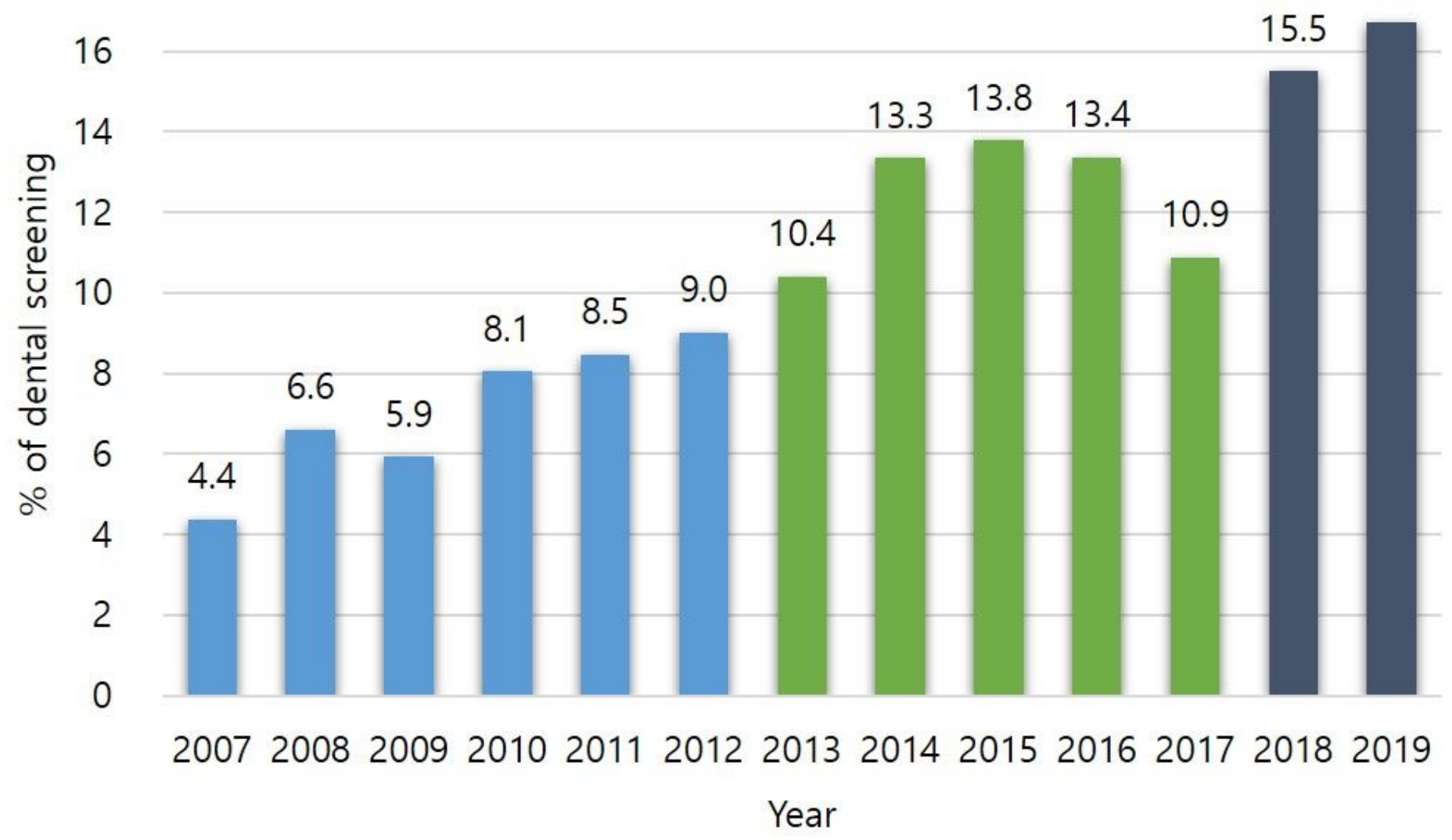

Figure 1

The trend of dental check-up before BMA treatment since 2007. 


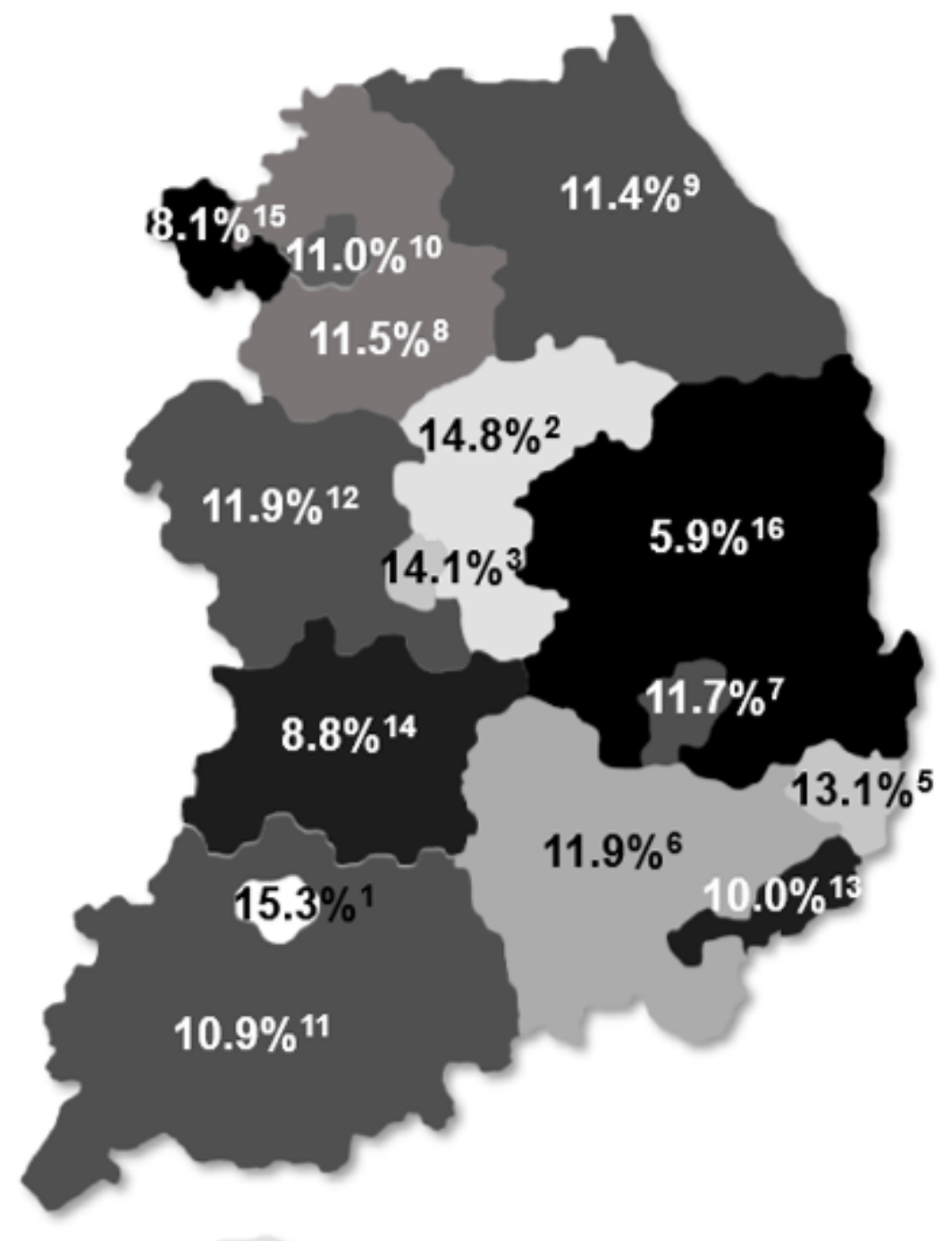

$13.8 \%^{4}$
1 Gwangju

2 Chungcheongbukdo

3 Daejeon

4 Jeju

5 Ulsan

6 Gyeongsangnamdo

7 Daegu

8 Gyeonggido

9 Gangwondo

10 Seoul

11 Jeollanamdo

12 Chungcheongnamdo

13 Busan

14 Jeollabukdo

15 Inchon 16 Gyeongsangbukdo

\section{Figure 2}

Dental check-up status before BMA treatment by region. 


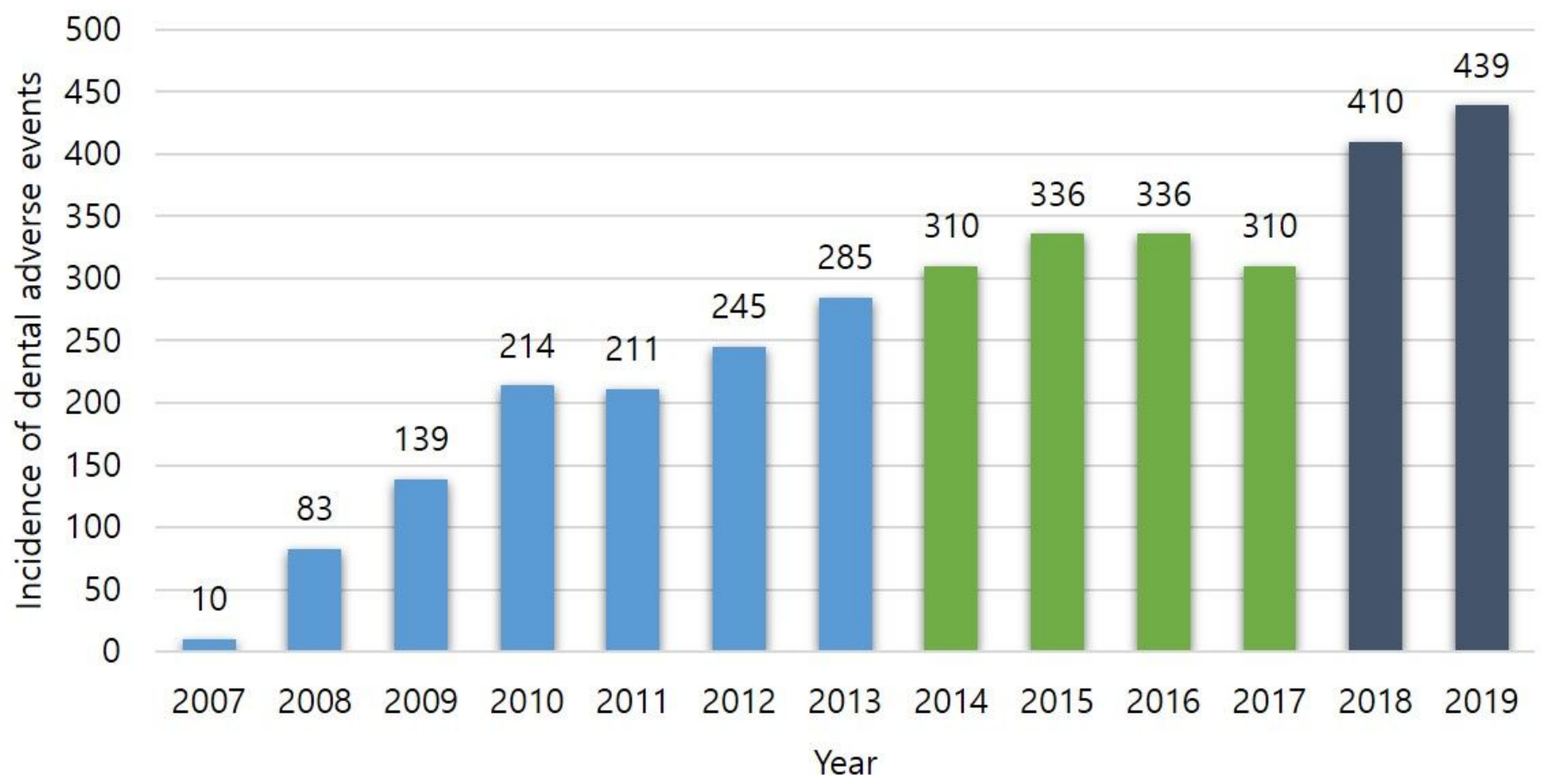

Figure 3

Incidence of adverse dental events after BMA treatment.

\section{Supplementary Files}

This is a list of supplementary files associated with this preprint. Click to download.

- SupplementTables.docx 